Publ. RIMS, Kyoto Univ.

12 Suppl. (1977), 465-468.

\title{
The Structure of Local Solutions of Partial Differential Equations of the Fuchsian Type
}

\author{
by
}

Hidetoshi TAHARA*

Linear partial differential equations with regular singularity along a hypersurface were studied by several authors, say, Hasegawa [10][11], Baouendi-Goulaouic [3] [4], Alinhac [1][2], Froim [7][8][9], DelacheLeray [6], Kashiwara-Oshima [13], Tsuno [15], etc $\cdots$ in various problems. In this note, we consider the hyperfunction solutions of certain type equations with regular singularity. The details of this note will be published in [14] anywhere else.

Let $(t, z) \in \boldsymbol{C} \times \boldsymbol{C}^{n}$ and let

$$
\begin{aligned}
P\left(t, z, D_{t}, D_{z}\right)= & t^{k} D_{t}{ }^{m}+P_{1}\left(t, z, D_{z}\right) t^{k-1} D_{t}{ }^{m-1}+\cdots \\
& +P_{k}\left(t, z, D_{z}\right) D_{t}{ }^{m-k}+\cdots+P_{m}\left(t, z, D_{z}\right)
\end{aligned}
$$

be a linear differential operator whose coefficients are holomorphic functions defined in a neighbourhood of the origin such that

(A-i) $\quad 0 \leqq k \leqq m$

(A-ii) $\quad$ order of $P_{j}\left(t, z, D_{z}\right) \leqq j$ for $1 \leqq j \leqq m$

(A-iii) order of $P_{j}\left(0, z, D_{z}\right) \leqq 0$ for $1 \leqq j \leqq k$.

Then $P$ is said of the Fuchsian type with weight m-k with respect to $t$ (by [3]). By the condition (A-iii), $P_{j}\left(0, z, D_{z}\right)$ is a function. We set $P_{j}\left(0, z, D_{z}\right)=a_{j}(z)$ for $1 \leqq j \leqq k$. Then the indicial equation associated with $P$ is defined by

$$
\mathscr{C}(\lambda, z)=\lambda(\lambda-1) \cdots(\lambda-m+1)+a_{1}(z) \lambda(\lambda-1) \cdots(\lambda-m+2)
$$

Received August 28, 1976.

* Department of Mathematics, Faculty of Science and Technology, Sophia University, 7 Kioicho, Chiyoda-ku, Tokyo, Japan. 


$$
+\cdots+a_{k}(z) \lambda(\lambda-1) \cdots(\lambda-m+k+1) .
$$

The roots, that we call the characteristic exponents of $P$, are denoted by $\lambda=0,1, \cdots, m-k-1, \rho_{1}(z), \cdots, \rho_{k}(z)$. They are functions of $z$.

We set

$\widetilde{\mathcal{O}}=$ the set of all the germs of multivalued holomorphic functions on $C \times C^{n} \backslash\{t=0\}$ at the origin.

Then we have the next theorem.

Theorem 1. Assume that $\rho_{i}(0), \rho_{i}(0)-\rho_{j}(0) \notin \mathbb{Z}$ holds for $1 \leqq i$ $\neq j \leqq k$. Then the equation $P u=f$ is always solvable in $\widetilde{\mathcal{O}}$. Moreover there exist holomorphic functions $K_{i}(t, z, w) \quad(0 \leqq i \leqq m-k-1), L_{j}(t$, $z, w)(1 \leqq j \leqq k)$ on

$$
\begin{aligned}
& U_{\varepsilon}=\left\{(t, z, w) \in C \times C^{n} \times C^{n} ;|t|,|z|,|w|<\varepsilon,\right. \\
& \left.\qquad|t|<M\left|z_{i}-w_{i}\right|^{s}, i=1, \cdots, n\right\} \\
& s=\min (m, k+1), M=\text { constant }
\end{aligned}
$$

which satisfy the following conditions:

(1) For any holomorphic functions $\varphi_{i}(w), \psi_{j}(w)$ at the origin, we set

$$
\begin{aligned}
u(t, z)= & \sum_{i=0}^{m-k-1} \oint K_{i}(t, z, w) t^{i} \varphi_{i}(w) d w \\
& +\sum_{j=1}^{k} \oint L_{j}(t, z, w) t^{\rho_{j}(w)} \psi_{j}(w) d w
\end{aligned}
$$

Then $u(t, z)$ is a solution of the equation $P u=0$ in $\widetilde{\mathcal{O}}$.

(2) If $u(t, z) \in \widetilde{\mathcal{O}}$ and $P u=0$ holds, then $u(t, z)$ is uniquely expressed in the form (1).

Next, we consider the equation in the real domain and investigate the structure of hyperfunction solutions. Let $(t, x) \in \boldsymbol{R} \times \boldsymbol{R}^{n}$ and let $P\left(t, x, D_{t}, D_{x}\right)$ be of the Fuchsian type with weight $m-k$ with respect to $t$. Moreover we assume the following conditions on $P$ :

(A-iv) $\sigma_{m}(P)$ has the form: $\sigma_{m}(P)(t, x, \tau, \xi)=t^{k} p_{m}(t, x, \tau, \xi)$

(A-v) All the roots $\tau(t, x, \xi)$ of the equation $p_{m}(t, x, \tau, \xi)=0$ are real, when $t, x, \xi$ are real (near the origin). 
Then we say that $P$ is a Funchsian hyperbolic operator with respect to $t$. Note that if $k=0$, then $P$ is nothing but a weakly hyperbolic operator in the direction $d t([5])$.

Under these assumptions, we can give the meaning as hyperfunctions to the above $K_{i}(t, z, w), L_{j}(t, z, w)$ in Theorem 1 . We also denote these hyperfunctions by $K_{i}(t, x, y), L_{j}(t, x, y)$ respectively. then $K_{i}, L_{j}$ satisfy the following conditions:

Supp $K_{i}, L_{j} \subset\left\{(t, x, y) ;|x-y| \leqq M|t|^{1 / s}\right\}$

$$
\begin{gathered}
S-S\left(K_{i}\right),\left(L_{j}\right) \subset\left\{(t, x, y, \sqrt{-1}(\tau, \xi, \eta) \infty) ;|x-y| \leqq M|t|^{1 / s},\right. \\
\left.|\tau| \leqq M|\xi|,|\xi+\eta| \leqq M|\xi||t|^{1 / s}\right\} .
\end{gathered}
$$

Using these hyperfunctions, we have the next theorem.

Theorem 2. Assume that $\rho_{i}(0), \rho_{i}(0)-\rho_{j}(0) \notin \mathbb{Z}$ holds for $1 \leqq i$ $\neq j \leqq k$. Then the equation $P u=f$ is always solvable in $\mathscr{B}$ (where $\mathscr{B}$ is the stalk of the sheaf of hyperfunctions at the origin). Moreover the above $K_{i}(t, x, y)(0 \leqq i \leqq m-k-1), L_{j}(t, x, y)(1 \leqq j \leqq k)$ satisfy the following conditions:

(1) For any hyperfunctions $\varphi_{i}(y), \psi_{j}^{ \pm}(y)$ at the origin, we set

$$
\begin{aligned}
u(t, x)= & \sum_{i=0}^{m-k-1} \int K_{i}(t, x, y) t^{i} \varphi_{i}(y) d y \\
& +\sum_{j=1}^{k} \sum_{ \pm} \int L_{j}(t, x, y)(t \pm i 0)^{\rho_{j}(y)} \psi_{j}^{ \pm}(y) d y
\end{aligned}
$$

or

$$
\begin{aligned}
u(t, x)= & \sum_{i=0}^{m-k-1} \int K_{i}(t, x, y) t^{i} \varphi_{i}(y) d y \\
& +\sum_{j=1}^{k} \sum_{ \pm} \int L_{j}(t, x, y) t_{ \pm}^{\rho_{j}(y)} \psi_{j}^{ \pm}(y) d y
\end{aligned}
$$

Then $u(t, x)$ is a solution of the equation $P u=0$ in $\mathcal{B}$.

(2) If $u(t, x) \in \mathscr{B}$ and $P u=0$ holds, then $u(t, x)$ is uniquely expressed in the form (1).

\section{References}

[1] Alinhac, S., Problèmes de Cauchy pour des opérateurs singuliers, Bull. Soc. Math. France, 102 (1974), 289-315. 
[2] Alinhac, S., Problèmes hyperboliques singuliers, Seminaire Goulaouic-Schwartz, 1973-1974.

[3] Baouendi, M. S. and Goulaouic, C., Cauchy problems with characteristic initial hypersurface, Comm. Pure. Appl. Math. 26 (1973), 455-475.

[4] Baouendi, M. S. and Goulaouic, C., Cauchy problems with multiple characteristics in spaces of regular distributions, Uspehi Mat. Nauk, 29-2 (1974), 70-76 (in Russian)

[5] Bony, J. M. and Schapira, P., Solutions hyperfonctions du problème de Cauchy, Lecture Notes in Math. No. 287, Springer (1973), 82-98.

[6] Delache, S. and Leray, J., Calcul de la solution élémentaire de l'opérateur d'EulerPoisson-Darboux et de l'opérateur de Tricomi-Clairaut, hyperbolique, d'order 2, Bull. Soc. Math. France, 99 (1971), 313-336.

[7] Froim, V. Kh., Linear scalar partial differential equations with regular singularities on a hypersurface, Differentsial'nye Uravneniya, 9-3 (1973), 533-541 (in Russian).

[8] Froim, V. Kh., The representation of analytic solutions of certain partial differential equations with coefficients having pole-type singularities with respect to one argument, Differentsial'nye Uravneniya, 7-1 (1971), 142-156 (in Russian).

[9] Froim, V. Kh., Linear partial differential equations whose coefficients have pole type singularities in one argument only, Siberian Math. J., 11-3 (1970), 625-647 (in Russian).

I. [10] Hasegawa, Y., On the initial-value problems with data on a double characteristic, J. Math. Kyoto Univ. 11-2 (1971), 357-372.

[11] Hasegawa, Y., On the initial-value problems with data on a characteristic hypersurface, J. Math. Kyoto Univ. 13-3 (1973), 579-593.

[12] Kashiwara, M. and Kawai, T., Micro-hyperbolic pseudo-differential operators I, $J$. Math. Soc. Japan, 27 (1975), 359-404.

[13] Kashiwara, M. and Oshima, T., Systems of differential equations with regular singularity and their boundary value problems, to appear.

[14] Tahara, H., Fuchsian type equations and Fuchsian hyperbolic equations, to appear.

[15] Tsuno, Y., On the prolongation of local holomorphic solutions of partial differential equations, III, equations of the Fuchsian type, to appear in J. Math. Soc. Japan. 